\title{
Functional Imaging of Cortical Responses in Expert and Novice Badminton Players while Predicting the Direction of a Badminton
} Stroke

\author{
$\underline{\text { MJ Wright, DT Bishop, }}$ RC Jackson, $\underline{\text { B Abernethy }}$
}

INTRODUCTION: Abernethy et al. (2008) showed that expert badminton players can use the kinematic information of the opponent's body movement to predict the direction of a stroke. The purpose of the present study was to compare the brain activity of expert and novice badminton players while carrying out a similar anticipation task. METHODS: In a block design fMRI study, participants viewed $2 \mathrm{~s}$ video displays of an opposing player, and pressed a button to indicate which of four possible court positions a stroke was directed. Individual trials were occluded either $80 \mathrm{~ms}$ before or $80 \mathrm{~ms}$ after the racquet-shuttle contact. Early- and late-occluded stimuli were presented in different blocks, as were 'no-shot' control video clips of the same opposing player. A second version of the task (in a separate fMRI run) used point-light stimuli, which preserved the purely kinematic cues of the opponent's action. Three groups of participants were tested: experts (12 national/international level badminton players), intermediates (11 club players) and novices (13 occasional/inexperienced players). For each participant, the magnitude of responses was quantified as percent signal change within a set of 9 pairs (left and right hemisphere) of $8 \mathrm{~mm}$ spherical regions of interest, whose location was determined on the basis of previous studies (e.g. Wright \& Jackson, 2007). RESULTS: Behavioral data showed superior accuracy by experts in both occlusion conditions, and for full video and point-light conditions. BOLD fMRI t-contrasts for all four experimental conditions relative to controls showed considerable overlap. However, a second-level analysis of variance revealed significant differences between expert, intermediate and novice groups in a number of brain regions of interest. Main effects of group (stronger activations in experts) were found in supplementary eye field (SEF: $p<0.005$ ), supplementary motor area (SMA: $p<0.01$ ), in two regions of inferior frontal gyrus: BA45 $(p<0.05)$ and BA47 $(p<0.05)$, and in inferior parietal lobule $(p<0.01)$. In the supplementary eye field (SEF: $\mathrm{p}<0.01$ ), and in inferior frontal gyrus (BA45: $p<0.005$; BA47: $p<0.005$ ) there was also a significant interaction between expertise and level of occlusion, such that experts showed proportionally greater activation in the pre-contact occlusion condition. The same pattern was not however found in V5/MT, where expert and novice responses were not significantly different. Full video and point-light versions of the task yielded very similar results overall for both experts and novices.

CONCLUSIONS: For experts, the pre-contact occlusion stimuli were strongly differentiated from control stimuli in SEF and in inferior frontal gyrus. This is consistent with these regions' involvement in experts' superior ability to analyse or model the kinematic information of an opponent's body movement. 